J. Nonlinear Var. Anal. 3 (2019), No. 1, pp. 5-18

Available online at http://jnva.biemdas.com

https://doi.org/10.23952/jnva.3.2019.1.02

\title{
STRONG CONVERGENCE FOR A MODIFIED FORWARD-BACKWARD SPLITTING METHOD IN BANACH SPACES
}

\author{
YASUNORI KIMURA ${ }^{1, *}$, KAZUHIDE NAKAJO $^{2}$ \\ ${ }^{1}$ Department of Information Science, Toho University, Miyama, Funabashi, Chiba 274-8510, Japan \\ ${ }^{2}$ Sundai Preparatory School, Surugadai, Kanda, Chiyoda-ku, Tokyo 101-8313, Japan \\ Dedicated to Professor Wataru Takahashi on the occasion of his 75th birthday
}

\begin{abstract}
We propose a modified forward-backward splitting method and prove a new strong convergence theorem of solutions to a zero problem of the sum of a monotone operator and an inverse-strongly-monotone operator in a real 2-uniformly convex and uniformly smooth Banach space. Some new results for variational inequality problems and monotone inclusions are obtained.
\end{abstract}

Keywords. Sum of maximal monotone operators; Forward-backward splitting method; Variational inequality; Inverse strongly monotone operators, 2-uniformly convex Banach space.

2010 Mathematics Subject Classification. 47H05, 47H14, 49J40.

\section{INTRODUCTION}

Let $E$ be a real Banach space with norm $\|\cdot\|$ and let $E^{*}$ be the dual of $E$. For $x \in E$ and $x^{*} \in E^{*}$, let $\left\langle x, x^{*}\right\rangle$ be the value of $x^{*}$ at $x$. Let $A \subset E \times E^{*}$ and $B \subset E \times E^{*}$ be maximal monotone operators such that $A+B$ is maximal monotone and $(A+B)^{-1} 0 \neq \emptyset$. Finding an element of $(A+B)^{-1} 0$ is so general that it concludes a number of important problems such as convex minimization problems, variational inequality problems, complementary problems, and others. In a real Hilbert space $H$, Passty [31] and Lions and Mercier [20] introduced the following forward-backward splitting method as one of the methods of finding an element of $(A+B)^{-1} 0$ :

$$
x_{1}=x \in D(B), \quad x_{n+1}=J_{\lambda_{n}}^{A}\left(x_{n}-\lambda_{n} w_{n}\right)
$$

for every $n \in \mathbb{N}$, where $D(B) \subset H$ is the domain of $B, w_{n} \in B x_{n},\left\{\lambda_{n}\right\} \subset(0, \infty)$, and $J_{\lambda_{n}}^{A}$ is the resolvent of $A$. Later, the splitting method was widely studied by Gabay [12], Chen and Rockafellar [9], Moudafi and Théra [26] and Tseng [41].

Let $\alpha>0$. A single valued operator $B: H \rightarrow H$ is said to be $\alpha$-inverse-strongly-monotone if

$$
\langle x-y, B x-B y\rangle \geq \alpha\|B x-B y\|^{2}
$$

for all $x, y \in H$; see [5, 11, 21, 45]. If $\alpha=1, B$ is called a firmly nonexpansive mapping. Gabay [12] proved that the sequence $\left\{x_{n}\right\}$ generated by (1.1) converges weakly to some $z \in(A+B)^{-1} 0$ when $B$ is

${ }^{*}$ Corresponding author.

E-mail addresses: yasunori@is.sci.toho-u.ac.jp (Y. Kimura), knkjyna@jcom.zaq.ne.jp (K. Nakajo).

Received August 30, 2018; Accepted January 27, 2019.

(C) 2019 Journal of Nonlinear and Variational Analysis 
$\alpha$-inverse-strongly-monotone and $\lambda_{n}=\lambda$ (constant) with $0<\lambda<2 \alpha$. Later, many researchers studied the weak convergence in a real Hilbert space; see $[4,27,28,37]$ and references therein. Nakajo, Shimoji and Takahashi [30] considered the following Halpern's type iteration [13]:

$$
x_{1}=x \in H, \quad x_{n+1}=\gamma_{n} x+\left(1-\gamma_{n}\right) J_{\lambda_{n}}^{A}\left(x_{n}-\lambda_{n} B x_{n}\right)
$$

for all $n \in \mathbb{N}$, where $B$ is $\alpha$-inverse-strongly-monotone, $\left\{\lambda_{n}\right\} \subset[a, 2 \alpha]$ for some $a \in(0,2 \alpha)$ with $\sum_{n=1}^{\infty} \mid \lambda_{n}-$ $\lambda_{n+1} \mid<\infty$ and $\left\{\gamma_{n}\right\} \subset[0,1)$ such that $\lim _{n \rightarrow \infty} \gamma_{n}=0, \prod_{n=1}^{\infty}\left(1-\gamma_{n}\right)=0$ and $\sum_{n=1}^{\infty}\left|\gamma_{n}-\gamma_{n+1}\right|<\infty$. They proved $\left\{x_{n}\right\}$ converges strongly to $P_{(A+B)^{-1} 0} x$, where $P_{(A+B)^{-1} 0}$ is the metric projection of $H$ onto $(A+B)^{-1} 0$. Later, strong convergence by the viscosity approximation [25] which extends that by Halpern's type iteration was studied by many researchers ([7, 10, 32, 36] and references therein) in a real Hilbert space. On the other hand, strong convergence by the hybrid method [14] and shrinking projection method [40] were researched by several authors $([28,29,43]$ and references therein) in a real Hilbert space.

In this paper, we consider a new iteration scheme and study its strong convergence in a real Banach space. Let $C$ be a nonempty closed convex subset of a real 2-uniformly convex and uniformly smooth Banach space $E$. Let $A \subset E \times E^{*}$ be a maximal monotone operator, and let $B$ be an inverse-stronglymonotone operator of $C$ into $E^{*}$, that is, there exists $\alpha>0$ such that $\langle x-y, B x-B y\rangle \geq \alpha\|B x-B y\|^{2}$ holds for every $x, y \in C$. Suppose that $F=(A+B)^{-1} 0 \neq \emptyset$. We propose the following modified forwardbackward splitting method:

$$
x_{1} \in C, \quad x_{n+1}=\Pi_{C} J_{\lambda_{n}}^{A} J^{-1}\left(\gamma_{n} J u+\left(1-\gamma_{n}\right) J x_{n}-\lambda_{n} B x_{n}\right)
$$

for each $n \in \mathbb{N}$, where $u \in E, \Pi_{C}$ is the generalized projection of $E$ onto $C,\left\{\lambda_{n}\right\} \subset(0, \infty), J_{\lambda_{n}}^{A}$ is the resolvent of $A, J$ is the duality mapping of $E$, and $\left\{\gamma_{n}\right\} \subset(0,1]$ such that $\gamma_{n} \rightarrow 0$ and $\sum_{n=1}^{\infty} \gamma_{n}=\infty$. We prove that $\left\{x_{n}\right\}$ converges strongly to $\Pi_{F} u$ under some conditions on $\left\{\lambda_{n}\right\}$. Further, we obtain new results for variational inequality problems and monotone inclusions.

\section{PRELIMINARIES}

Throughout this paper, we denote by $\mathbb{N}$ and by $\mathbb{R}$ the set of all positive integers and the set of all real numbers, respectively. We use $x_{n} \rightarrow x$ to indicate that a sequence $\left\{x_{n}\right\}$ converges weakly to $x$ and $x_{n} \rightarrow x$ will symbolize strong convergence. We define the modulus of convexity $\delta_{E}$ of $E$ as follows: $\delta_{E}$ is a function of $[0,2]$ into $[0,1]$ such that

$$
\delta_{E}(\varepsilon)=\inf \{1-\|x+y\| / 2: x, y \in E,\|x\|=1,\|y\|=1,\|x-y\| \geq \varepsilon\}
$$

for every $\varepsilon \in[0,2]$. $E$ is said to be uniformly convex if $\delta_{E}(\varepsilon)>0$ for each $\varepsilon>0$. For $p>1$, we say $E$ is $p$-uniformly convex if there exists a constant $c>0$ such that $\delta_{E}(\varepsilon) \geq c \varepsilon^{p}$ for every $\varepsilon \in[0,2]$. It is obvious that a $p$-uniformly convex Banach space is uniformly convex. $E$ is said to be strictly convex if $\|x+y\| / 2<1$ for all $x, y \in E$ with $\|x\|=\|y\|=1$ and $x \neq y$. We know that a uniformly convex Banach space is strictly convex and reflexive. The duality mapping $J: E \rightarrow 2^{E^{*}}$ of $E$ is defined by

$$
J(x)=\left\{f \in E^{*}:\langle x, f\rangle=\|x\|^{2}=\|f\|^{2}\right\}
$$


for every $x \in E$. It is also known that if $E$ is strictly convex and reflexive, then, the duality mapping $J$ of $E$ is bijective, and $J^{-1}: E^{*} \rightarrow 2^{E}$ is the duality mapping of $E^{*} . E$ is said to be smooth if the limit

$$
\lim _{t \rightarrow 0} \frac{\|x+t y\|-\|x\|}{t}
$$

exists for every $x, y \in S(E)$, where $S(E)=\{x \in E:\|x\|=1\}$. E is said to be uniformly smooth if limit (2.1) is attained uniformly for $(x, y)$ in $S(E) \times S(E)$. It is known that $E$ is uniformly smooth if and only if $E^{*}$ is uniformly convex. We know that the duality mapping $J$ of $E$ is single-valued if and only if $E$ is smooth. We also know that if $E$ is uniformly smooth, then the duality mapping $J$ of $E$ is uniformly continuous on bounded subsets of $E$; see $[38,39]$ for more details.

The following was proved by $\mathrm{Xu}$ [42]; see also [44].

Theorem 2.1. Let $E$ be a smooth Banach space. Then, $E$ is 2-uniformly convex if and only if there exists a constant $c>0$ such that for each $x, y \in E,\|x+y\|^{2} \geq\|x\|^{2}+2\langle y, J x\rangle+c\|y\|^{2}$ holds.

Remark 2.1. In a real Hilbert space, we can choose $c=1$.

Let $E$ be a smooth Banach space. The function $\phi: E \times E \rightarrow \mathbb{R}$ is defined by

$$
\phi(y, x)=\|y\|^{2}-2\langle y, J x\rangle+\|x\|^{2}
$$

for every $x, y \in E$. It is obvious that $(\|y\|-\|x\|)^{2} \leq \phi(y, x) \leq(\|y\|+\|x\|)^{2}$ for each $x, y \in E$ and $\phi(z, x)+$ $\phi(x, y)=\phi(z, y)+2\langle x-z, J x-J y\rangle$ for all $x, y, z \in E$. We also know that if $E$ is strictly convex and smooth, then, for $x, y \in E, \phi(y, x)=0$ if and only if $x=y$; see [23].

We have the following result by Theorem 2.1; see also [17].

Lemma 2.1. Let $E$ be a 2-uniformly convex and smooth Banach space. Then, for each $x, y \in E, \phi(x, y) \geq$ $c\|x-y\|^{2}$ holds, where $c$ is the constant in Theorem 2.1.

Let $C$ be a nonempty closed convex subset of a strictly convex, reflexive and smooth Banach space $E$ and let $x \in E$. Then, there exists a unique element $x_{0} \in C$ such that

$$
\phi\left(x_{0}, x\right)=\inf _{y \in C} \phi(y, x) .
$$

We denote $x_{0}$ by $\Pi_{C} x$ and call $\Pi_{C}$ the generalized projection of $E$ onto $C$; see $[1,2,16]$. We have the following well-known result $[1,2,16]$ for the generalized projection.

Lemma 2.2. Let $C$ be a nonempty convex subset of a smooth Banach space $E, x \in E$ and $x_{0} \in C$. Then, $\phi\left(x_{0}, x\right)=\inf _{y \in C} \phi(y, x)$ if and only if $\left\langle x_{0}-z, J x-J x_{0}\right\rangle \geq 0$ for every $z \in C$, or equivalently, $\phi(z, x) \geq$ $\phi\left(z, x_{0}\right)+\phi\left(x_{0}, x\right)$ for all $z \in C$.

An operator $A \subset E \times E^{*}$ is said to be monotone if $\left\langle x-y, x^{*}-y^{*}\right\rangle \geq 0$ for every $\left(x, x^{*}\right),\left(y, y^{*}\right) \in A$. A monotone operator $A$ is said to be maximal if the graph of $A$ is not properly contained in the graph of any other monotone operator. We know that a monotone operator $A$ is maximal if and only if for $\left(u, u^{*}\right) \in E \times E^{*},\left\langle x-u, x^{*}-u^{*}\right\rangle \geq 0$ for every $\left(x, x^{*}\right) \in A$ implies $\left(u, u^{*}\right) \in A$. Rockafellar [35] proved the following result; see also [8].

Theorem 2.2. Let $E$ be a strictly convex, reflexive and smooth Banach space and let $A \subset E \times E^{*}$ be a monotone operator. Then, $A$ is maximal if and only if $R(J+r A)=E^{*}$, for all $r>0$, where $R(J+r A)$ is the range of $J+r A$. 
Let $E$ be a strictly convex, reflexive and smooth Banach space and let $A \subset E \times E^{*}$ be a maximal monotone operator. By Theorem 2.2 and strict convexity of $E$, for any $x \in E$ and $r>0$, there exists a unique element $x_{r} \in D(A)$ such that

$$
J(x) \in J\left(x_{r}\right)+r A x_{r},
$$

where $D(A)$ is the domain of $A$. We define $J_{r}$ by $J_{r} x=x_{r}$ for every $x \in E$ and $r>0$ and such $J_{r}$ is called the resolvent of $A$; see[6,39] for more details.

Let $f: E \rightarrow(-\infty, \infty]$ be a proper, lower semicontinuous and convex function. Then, it is known that the subdifferential $\partial f$ of $f$ defined by

$$
\partial f(x)=\left\{x^{*} \in E^{*}: f(y) \geq f(x)+\left\langle y-x, x^{*}\right\rangle, \forall y \in E\right\}
$$

for $x \in E$ is a maximal monotone operator; see [33,34].

A function $\tau: \mathbb{N} \rightarrow \mathbb{N}$ is said to be eventually increasing if $\lim _{n \rightarrow \infty} \tau(n)=\infty$ and $\tau(n) \leq \tau(n+1)$ for all $n \in \mathbb{N}$. The following was proved by Aoyama, Kimura and Kohsaka [3, Lemma 3.4]; see also [22, Lemma 3.1].

Lemma 2.3. Let $\left\{\xi_{n}\right\}$ be a sequence of nonnegative real numbers which is not convergent. Then there exist $n_{0} \in \mathbb{N}$ and an eventually increasing function $\tau$ such that $\xi_{\tau(n)} \leq \xi_{\tau(n)+1}$ for all $n \in \mathbb{N}$ and $\xi_{n} \leq$ $\xi_{\tau(n)+1}$ for every $n \geq n_{0}$.

\section{MAIN RESUltS}

We first prove the following important lemmas.

Lemma 3.1. Let $C$ be a nonempty closed convex subset of a strictly convex, reflexive and smooth Banach space $E, A \subset E \times E^{*}$ a maximal monotone operator and $B: C \rightarrow E^{*}$ such that $(A+B)^{-1} 0 \neq \emptyset$. Suppose that there exists a real number $\alpha>0$ with $\langle x-z, B x-B z\rangle \geq \alpha\|B x-B z\|^{2}$ for all $x \in C$ and $z \in(A+B)^{-1} 0$. Let $T_{\lambda} x=J_{\lambda}^{A} J^{-1}(J x-\lambda B x)$ for $\lambda>0$ and $x \in C$, where $J_{\lambda}^{A}$ is the resolvent of $A$. Then, the following hold:

(i) $F\left(T_{\lambda}\right)=(A+B)^{-1} 0$ for all $\lambda>0$, where $F\left(T_{\lambda}\right)$ is the set of all fixed points of $T_{\lambda}$;

(ii) if $E$ is 2-uniformly convex, $\phi\left(z, T_{\lambda} x\right) \leq \phi(z, x)-(c-\lambda \beta)\left\|x-T_{\lambda} x\right\|^{2}-\lambda(2 \alpha-1 / \beta)\|B x-B z\|^{2}$ holds for every $\lambda, \beta>0, x \in C$ and $z \in(A+B)^{-1} 0$, where $c$ is the constant in Theorem 2.1;

(iii) if $E$ is 2-uniformly convex, $(A+B)^{-1} 0$ is closed and convex.

Proof. (i) Let $z \in(A+B)^{-1} 0$. Then we have $-B z \in A z$ and it follows that

$$
J z-\lambda B z \in J z+\lambda A z
$$

which is equivalent to $J_{\lambda}^{A} J^{-1}(J z-\lambda B z)=z$. Thus we have $z \in F\left(T_{\lambda}\right)$, The implication of the opposite direction is also straightforward. Hence we have $F\left(T_{\lambda}\right)=(A+B)^{-1} 0$.

(ii) Let $\lambda, \beta>0, x \in C, z \in(A+B)^{-1} 0$ and $y=T_{\lambda} x$. We have

$$
\phi(z, y)=\phi(z, x)-\phi(y, x)+2\langle y-z, J y-J x\rangle .
$$

Since $z \in(A+B)^{-1} 0$ and $y=J_{\lambda}^{A} J^{-1}(J x-\lambda B x)$, we get

$$
\left\langle y-z, \frac{1}{\lambda}(J x-J y)-B x+B z\right\rangle \geq 0,
$$


which implies

$$
\langle y-z, J x-J y\rangle \geq \lambda\langle y-z, B x-B z\rangle
$$

So, we obtain

$$
\begin{aligned}
\phi(z, y) & \leq \phi(z, x)-\phi(y, x)-2 \lambda\langle y-z, B x-B z\rangle \\
& =\phi(z, x)-\phi(y, x)-2 \lambda\langle y-x, B x-B z\rangle-2 \lambda\langle x-z, B x-B z\rangle \\
& \leq \phi(z, x)-\phi(y, x)-2 \lambda\langle y-x, B x-B z\rangle-2 \lambda \alpha\|B x-B z\|^{2} .
\end{aligned}
$$

By Lemma 2.1,

$$
\phi(z, y) \leq \phi(z, x)-c\|y-x\|^{2}+2 \lambda\|y-x\|\|B x-B z\|-2 \lambda \alpha\|B x-B z\|^{2} .
$$

Since $\|y-x\|\|B x-B z\| \leq \frac{\beta}{2}\|y-x\|^{2}+\frac{1}{2 \beta}\|B x-B z\|^{2}$, we have

$$
\phi(z, y) \leq \phi(z, x)-(c-\lambda \beta)\|y-x\|^{2}-\lambda\left(2 \alpha-\frac{1}{\beta}\right)\|B x-B z\|^{2} .
$$

(iii) For $\alpha>0$, there exists $\lambda_{0}>0$ with $c / \lambda_{0}>1 /(2 \alpha)$, where $c$ is the constant in Theorem 2.1. So, we can select $\beta>0$ such that $c / \lambda_{0}>\beta>1 /(2 \alpha)$. By (ii), we get $\phi\left(z, T_{\lambda_{0}} x\right) \leq \phi(z, x)$ for every $x \in C$ and $z \in(A+B)^{-1} 0$. From (i), $F\left(T_{\lambda_{0}}\right)=(A+B)^{-1} 0$. By the result in [23, 24], we have $F\left(T_{\lambda_{0}}\right)$ is closed and convex. Indeed, let $\left\{z_{n}\right\} \subset F\left(T_{\lambda_{0}}\right)$ such that $z_{n} \rightarrow z$. We have

$$
\phi\left(z_{n}, T_{\lambda_{0}} z\right) \leq \phi\left(z_{n}, z\right)
$$

for all $n \in \mathbb{N}$, which implies

$$
\phi\left(z, T_{\lambda_{0}} z\right) \leq 0 .
$$

So, we obtain $z \in F\left(T_{\lambda_{0}}\right)$, that is, $F\left(T_{\lambda_{0}}\right)$ is closed. Next, let $z_{1}, z_{2} \in F\left(T_{\lambda_{0}}\right), 0 \leq \gamma \leq 1$ and $x=\gamma z_{1}+(1-$ $\gamma) z_{2}$. It follows that

$$
\begin{aligned}
\phi\left(x, T_{\lambda_{0}} x\right) & =\|x\|^{2}-2\left\langle x, J\left(T_{\lambda_{0}} x\right)\right\rangle+\left\|T_{\lambda_{0}} x\right\|^{2} \\
& =\|x\|^{2}-2\left\langle\gamma z_{1}+(1-\gamma) z_{2}, J\left(T_{\lambda_{0}} x\right)\right\rangle+\left\|T_{\lambda_{0}} x\right\|^{2} \\
& =\|x\|^{2}+\gamma \phi\left(z_{1}, T_{\lambda_{0}} x\right)+(1-\gamma) \phi\left(z_{2}, T_{\lambda_{0}} x\right)-\gamma\left\|z_{1}\right\|^{2}-(1-\gamma)\left\|z_{2}\right\|^{2} \\
& \leq\|x\|^{2}+\gamma \phi\left(z_{1}, x\right)+(1-\gamma) \phi\left(z_{2}, x\right)-\gamma\left\|z_{1}\right\|^{2}-(1-\gamma)\left\|z_{2}\right\|^{2} \\
& =2\|x\|^{2}-2\left\langle\gamma z_{1}+(1-\gamma) z_{2}, J x\right\rangle \\
& =2\|x\|^{2}-2\|x\|^{2}=0 .
\end{aligned}
$$

Hence $x=T_{\lambda_{0}} x$, which implies $F\left(T_{\lambda_{0}}\right)$ is convex.

Lemma 3.2. Let $C$ be a nonempty closed convex subset of a strictly convex, reflexive and uniformly smooth Banach space $E$. Let $A \subset E \times E^{*}$ be a maximal monotone operator, $\alpha>0$ and $B: C \rightarrow E^{*}$ an $\alpha$-inverse-strongly-monotone operator such that $F=(A+B)^{-1} 0 \neq \emptyset$. Let $\left\{x_{n}\right\}$ be a bounded sequence in $C, u \in E$ and $y_{n}=J_{\lambda_{n}}^{A} J^{-1}\left(\gamma_{n} J u+\left(1-\gamma_{n}\right) J x_{n}-\lambda_{n} B x_{n}\right)$, where $\left\{\lambda_{n}\right\} \subset(0, \infty)$ with $\inf _{n \in \mathbb{N}} \lambda_{n}>0, J_{\lambda_{n}}^{A}$ is the resolvent of $A, J$ is the duality mapping of $E$ and $\left\{\gamma_{n}\right\} \subset(0,1]$ such that $\gamma_{n} \rightarrow 0$. If $\left\|x_{n}-y_{n}\right\| \rightarrow 0$ and $\left\|B x_{n}-B z\right\| \rightarrow 0$ for some $z \in F$, then $\omega_{w}\left(\left\{x_{n}\right\}\right) \subset F$, where $\omega_{w}\left(\left\{x_{n}\right\}\right)$ is the set of all weak cluster points of $\left\{x_{n}\right\}$. 
Proof. Let $\left(x, x^{*}\right) \in A$ and $\left\{x_{n_{i}}\right\} \subset\left\{x_{n}\right\}$ such that $x_{n_{i}} \rightarrow v$. Using

$$
\left\langle x_{n_{i}}-v, B x_{n_{i}}-B v\right\rangle \geq \alpha\left\|B x_{n_{i}}-B v\right\|^{2}
$$

and $\left\|B x_{n_{i}}-B z\right\| \rightarrow 0$, we have $\left\|B x_{n_{i}}-B v\right\| \rightarrow 0$. Since

$$
\frac{1}{\lambda_{n}}\left(J x_{n}-J y_{n}\right)-B x_{n}-\frac{\gamma_{n}}{\lambda_{n}}\left(J x_{n}-J u\right) \in A y_{n},
$$

we have

which implies

$$
\left\langle x-y_{n_{i}}, x^{*}-\frac{1}{\lambda_{n_{i}}}\left(J x_{n_{i}}-J y_{n_{i}}\right)+B x_{n_{i}}+\frac{\gamma_{n_{i}}}{\lambda_{n_{i}}}\left(J x_{n_{i}}-J u\right)\right\rangle \geq 0,
$$

$$
\begin{aligned}
& \left\langle x-y_{n_{i}}, x^{*}+B x_{n_{i}}\right\rangle \\
& \geq \frac{1}{\lambda_{n_{i}}}\left\langle x-y_{n_{i}}, J x_{n_{i}}-J y_{n_{i}}\right\rangle-\frac{\gamma_{n_{i}}}{\lambda_{n_{i}}}\left\langle x-y_{n_{i}}, J x_{n_{i}}-J u\right\rangle \\
& \geq-\frac{1}{\lambda_{n_{i}}}\left\|x-y_{n_{i}}\right\|\left\|J x_{n_{i}}-J y_{n_{i}}\right\|-\frac{\gamma_{n_{i}}}{\lambda_{n_{i}}}\left\|x-y_{n_{i}}\right\|\left\|J x_{n_{i}}-J u\right\|
\end{aligned}
$$

for all $i \in \mathbb{N}$. Since $\left\|y_{n_{i}}-x_{n_{i}}\right\| \rightarrow 0$ and $E$ is uniformly smooth, we have $\left\|J y_{n_{i}}-J x_{n_{i}}\right\| \rightarrow 0$. By $y_{n_{i}} \rightarrow v$, $\gamma_{n_{i}} \rightarrow 0$ and $\left\|B x_{n_{i}}-B v\right\| \rightarrow 0$, we obtain

$$
\left\langle x-v, x^{*}+B v\right\rangle \geq 0 .
$$

Since $A$ is maximal monotone, we have $-B v \in A v$, that is, $v \in(A+B)^{-1} 0=F$.

Now, we prove our main result.

Theorem 3.1. Let $C$ be a nonempty closed convex subset of a 2-uniformly convex and uniformly smooth Banach space $E$. Let $A$ be a maximal monotone operator in $E \times E^{*}, \alpha>0$ and $B$ an $\alpha$-inverse-stronglymonotone operator of $C$ into $E^{*}$ such that $F=(A+B)^{-1} 0 \neq \emptyset$. Let $u \in E$ and $\left\{x_{n}\right\}$ be a sequence generated by

$$
x_{1} \in C, \quad x_{n+1}=\Pi_{C} J_{\lambda_{n}}^{A} J^{-1}\left(\gamma_{n} J u+\left(1-\gamma_{n}\right) J x_{n}-\lambda_{n} B x_{n}\right)
$$

for each $n \in \mathbb{N}$, where $\Pi_{C}$ is the generalized projection of $E$ onto $C,\left\{\lambda_{n}\right\} \subset(0, \infty)$ and $\left\{\gamma_{n}\right\} \subset(0,1]$ such that $\gamma_{n} \rightarrow 0$ and $\sum_{n=1}^{\infty} \gamma_{n}=\infty$. Then, if $0<\inf _{n \in \mathbb{N}} \lambda_{n} \leq \sup _{n \in \mathbb{N}} \lambda_{n}<2 c \alpha$, where $c$ is the constant in Theorem 2.1, then $\left\{x_{n}\right\}$ converges strongly to $\Pi_{F} u$.

Proof. By Lemma 3.1 (iii), $F$ is closed and convex. Thus $\Pi_{F} u$ is well defined. Let $z \in F$ and

$$
y_{n}=J_{\lambda_{n}}^{A} J^{-1}\left(\gamma_{n} J u+\left(1-\gamma_{n}\right) J x_{n}-\lambda_{n} B x_{n}\right) .
$$

We have

$$
\phi\left(z, y_{n}\right)=\phi\left(z, x_{n}\right)-\phi\left(y_{n}, x_{n}\right)+2\left\langle y_{n}-z, J y_{n}-J x_{n}\right\rangle .
$$

Since $-B z \in A z$ and

$$
\frac{1}{\lambda_{n}}\left\{J x_{n}-J y_{n}-\lambda_{n} B x_{n}-\gamma_{n}\left(J x_{n}-J u\right)\right\} \in A y_{n},
$$

by the monotonicity of $A$ we get

$$
\left\langle y_{n}-z, \frac{1}{\lambda_{n}}\left(J x_{n}-J y_{n}\right)-\left(B x_{n}-B z\right)-\frac{\gamma_{n}}{\lambda_{n}}\left(J x_{n}-J u\right)\right\rangle \geq 0,
$$

which implies

$$
\left\langle y_{n}-z, J x_{n}-J y_{n}\right\rangle \geq \lambda_{n}\left\langle y_{n}-z, B x_{n}-B z\right\rangle+\gamma_{n}\left\langle y_{n}-z, J x_{n}-J u\right\rangle
$$


for all $n \in \mathbb{N}$. Thus we have

$$
\begin{aligned}
\phi\left(z, y_{n}\right) \leq & \phi\left(z, x_{n}\right)-\phi\left(y_{n}, x_{n}\right)-2 \lambda_{n}\left\langle y_{n}-z, B x_{n}-B z\right\rangle \\
& -2 \gamma_{n}\left\langle y_{n}-z, J x_{n}-J u\right\rangle
\end{aligned}
$$

for each $n \in \mathbb{N}$. Similarly in the calculation of Lemma 3.1 (ii), we get

$$
\begin{aligned}
\phi\left(z, y_{n}\right) \leq & \phi\left(z, x_{n}\right)-\left(c-\lambda_{n} \beta\right)\left\|x_{n}-y_{n}\right\|^{2} \\
& -\lambda_{n}(2 \alpha-1 / \beta)\left\|B x_{n}-B z\right\|^{2}-2 \gamma_{n}\left\langle y_{n}-z, J x_{n}-J u\right\rangle
\end{aligned}
$$

for every $\beta>0$ and $n \in \mathbb{N}$. Using $x_{n+1}=\Pi_{C} y_{n}$ and Lemma 2.2, we obtain

$$
\phi\left(x_{n+1}, y_{n}\right)+\phi\left(z, x_{n+1}\right) \leq \phi\left(z, y_{n}\right) .
$$

Therefore we have

$$
\begin{aligned}
\phi\left(z, x_{n+1}\right) \leq & \phi\left(z, x_{n}\right)-\left(c-\lambda_{n} \beta\right)\left\|x_{n}-y_{n}\right\|^{2} \\
& -\lambda_{n}(2 \alpha-1 / \beta)\left\|B x_{n}-B z\right\|^{2}-2 \gamma_{n}\left\langle y_{n}-z, J x_{n}-J u\right\rangle
\end{aligned}
$$

for each $\beta>0$ and $n \in \mathbb{N}$. Since

$$
0<\inf _{n \in \mathbb{N}} \lambda_{n} \leq \sup _{n \in \mathbb{N}} \lambda_{n}<2 c \alpha,
$$

there exists $\beta_{0}>0$ such that $\inf _{n \in \mathbb{N}}\left(c-\lambda_{n} \beta_{0}\right)>0$ and $\inf _{n \in \mathbb{N}} \lambda_{n}\left(2 \alpha-1 / \beta_{0}\right)>0$. Using

$$
2\left\langle y_{n}-z, J x_{n}-J u\right\rangle=\phi\left(y_{n}, u\right)+\phi\left(z, x_{n}\right)-\phi\left(y_{n}, x_{n}\right)-\phi(z, u)
$$

with (3.1), we have

$$
\begin{aligned}
\phi\left(z, x_{n+1}\right) \leq & \phi\left(z, x_{n}\right)-\left(1-\gamma_{n}\right) \phi\left(y_{n}, x_{n}\right)-2 \lambda_{n}\left\langle y_{n}-z, B x_{n}-B z\right\rangle \\
& -\gamma_{n}\left\{\phi\left(y_{n}, u\right)+\phi\left(z, x_{n}\right)-\phi(z, u)\right\} .
\end{aligned}
$$

Again, since $0<\inf _{n \in \mathbb{N}} \lambda_{n} \leq \sup _{n \in \mathbb{N}} \lambda_{n}<2 c \alpha$ and $\gamma_{n} \rightarrow 0$, there exist $N \in \mathbb{N}$ and $\delta_{0}>0$ such that $\inf _{n \geq N}\left\{\left(1-\gamma_{n}\right) c-\lambda_{n} \delta_{0}\right\}>0$ and $\inf _{n \in \mathbb{N}} \lambda_{n}\left(2 \alpha-1 / \delta_{0}\right)>0$. As in the calculation of Lemma 3.1 (ii), we obtain

$$
\begin{aligned}
\phi\left(z, x_{n+1}\right) \leq & \phi\left(z, x_{n}\right)-\left\{\left(1-\gamma_{n}\right) c-\lambda_{n} \delta_{0}\right\}\left\|y_{n}-x_{n}\right\|^{2} \\
& -\lambda_{n}\left(2 \alpha-1 / \delta_{0}\right)\left\|B x_{n}-B z\right\|^{2} \\
& -\gamma_{n}\left\{\phi\left(y_{n}, u\right)+\phi\left(z, x_{n}\right)-\phi(z, u)\right\}
\end{aligned}
$$

for all $n \in \mathbb{N}$. We prove $\left\{x_{n}\right\}$ is bounded. If $\left\{\phi\left(z, x_{n}\right)\right\}$ converges, it is trivial. If not so, by Lemma 2.3, there exist $n_{0} \in \mathbb{N}$ and an eventually increasing function $\tau$ such that $\phi\left(z, x_{\tau(n)}\right) \leq \phi\left(z, x_{\tau(n)+1}\right)$ for every $n \in \mathbb{N}$ and $\phi\left(z, x_{n}\right) \leq \phi\left(z, x_{\tau(n)+1}\right)$ for each $n \geq n_{0}$. Since

$$
\begin{gathered}
\inf _{\tau(n) \geq N}\left\{\left(1-\gamma_{\tau(n)}\right) c-\lambda_{\tau(n)} \delta_{0}\right\}>0, \\
\inf _{n \in \mathbb{N}} \lambda_{\tau(n)}\left(2 \alpha-1 / \delta_{0}\right)>0,
\end{gathered}
$$

$\phi\left(z, x_{\tau(n)}\right) \leq \phi\left(z, x_{\tau(n)+1}\right)$, and $\gamma_{\tau(n)}>0$, it follows from (3.5) that

$$
\phi\left(y_{\tau(n)}, u\right)+\phi\left(z, x_{\tau(n)}\right)-\phi(z, u)<0
$$

for all $n$ with $\tau(n) \geq N$, which implies that $\left\{x_{\tau(n)}\right\}$ and $\left\{y_{\tau(n)}\right\}$ are bounded. Further, we have

$$
\phi\left(z, x_{\tau(n)+1}\right) \leq \phi\left(z, x_{\tau(n)}\right)-\gamma_{\tau(n)}\left\{\phi\left(y_{\tau(n)}, u\right)+\phi\left(z, x_{\tau(n)}\right)-\phi(z, u)\right\}
$$


for every $n$ with $\tau(n) \geq N$. Thus we have $\left\{x_{\tau(n)+1}\right\}$ is bounded. Since $\phi\left(z, x_{n}\right) \leq \phi\left(z, x_{\tau(n)+1}\right)$ for each $n \geq n_{0}$, it follows that $\left\{x_{n}\right\}$ is bounded. From (3.2) with $\beta=\beta_{0}$, we obtain

$$
\phi\left(z, y_{n}\right) \leq \phi\left(z, x_{n}\right)-2 \gamma_{n}\left\langle y_{n}-z, J x_{n}-J u\right\rangle
$$

which implies

$$
\left(\|z\|-\left\|y_{n}\right\|\right)^{2} \leq\left(\|z\|+\left\|x_{n}\right\|\right)^{2}+2 \gamma_{n}\left(\left\|y_{n}\right\|+\|z\|\right)\left(\left\|x_{n}\right\|+\|u\|\right)
$$

for each $n \in \mathbb{N}$. Since $\left\{x_{n}\right\}$ is bounded, so is $\left\{y_{n}\right\}$.

Suppose that $\left\{\phi\left(\Pi_{F} u, x_{n}\right)\right\}$ is not convergent. By Lemma 2.3, there exist $n_{0} \in \mathbb{N}$ and an eventually increasing function $\tau$ such that $\phi\left(\Pi_{F} u, x_{\tau(n)}\right) \leq \phi\left(\Pi_{F} u, x_{\tau(n)+1}\right)$ for all $n \in \mathbb{N}$ and $\phi\left(\Pi_{F} u, x_{n}\right) \leq$ $\phi\left(\Pi_{F} u, x_{\tau(n)+1}\right)$ for every $n \geq n_{0}$. Since $\left\{x_{n}\right\}$ and $\left\{y_{n}\right\}$ are bounded, and $\gamma_{n} \rightarrow 0$, using (3.4) with $\beta=\beta_{0}$, we get

$$
\left\|y_{\tau(n)}-x_{\tau(n)}\right\| \rightarrow 0 \text { and }\left\|B x_{\tau(n)}-B \Pi_{F} u\right\| \rightarrow 0 .
$$

By Lemma 3.2, we get that $F$ includes $\omega_{w}\left(x_{\tau(n)}\right)$. By (3.4) with $\beta=\beta_{0}$, we have

$$
\begin{aligned}
0 & \leq\left(c-\lambda_{\tau(n)} \beta_{0}\right)\left\|x_{\tau(n)}-y_{\tau(n)}\right\|^{2}+\lambda_{\tau(n)}\left(2 \alpha-1 / \beta_{0}\right)\left\|B x_{\tau(n)}-B \Pi_{F} u\right\|^{2} \\
& \leq-2 \gamma_{\tau(n)}\left\langle y_{\tau(n)}-\Pi_{F} u, J x_{\tau(n)}-J u\right\rangle
\end{aligned}
$$

for every $n \in \mathbb{N}$. Since $\gamma_{\tau(n)}>0$,

$$
\left\langle y_{\tau(n)}-\Pi_{F} u, J x_{\tau(n)}-J u\right\rangle \leq 0
$$

for each $n \in \mathbb{N}$. On the other hand,

$$
\begin{aligned}
\left\langle y_{\tau(n)}-\Pi_{F} u, J x_{\tau(n)}-J u\right\rangle= & \left\langle y_{\tau(n)}-\Pi_{F} u, J x_{\tau(n)}-J y_{\tau(n)}\right\rangle \\
& +\left\langle y_{\tau(n)}-\Pi_{F} u, J y_{\tau(n)}-J \Pi_{F} u\right\rangle \\
& +\left\langle y_{\tau(n)}-\Pi_{F} u, J \Pi_{F} u-J u\right\rangle \\
\geq & \left\langle y_{\tau(n)}-\Pi_{F} u, J x_{\tau(n)}-J y_{\tau(n)}\right\rangle \\
& +\frac{1}{2} \phi\left(\Pi_{F} u, y_{\tau(n)}\right)+\left\langle y_{\tau(n)}-\Pi_{F} u, J \Pi_{F} u-J u\right\rangle .
\end{aligned}
$$

By (3.7), we get

$$
-\frac{1}{2} \phi\left(\Pi_{F} u, y_{\tau(n)}\right) \geq\left\langle y_{\tau(n)}-\Pi_{F} u, J x_{\tau(n)}-J y_{\tau(n)}\right\rangle+\left\langle y_{\tau(n)}-\Pi_{F} u, J \Pi_{F} u-J u\right\rangle
$$

for all $n \in \mathbb{N}$. So,

$$
\begin{aligned}
-\frac{1}{2} \limsup _{n \rightarrow \infty} \phi\left(\Pi_{F} u, y_{\tau(n)}\right) & \geq \liminf _{n \rightarrow \infty}\left\langle y_{\tau(n)}-\Pi_{F} u, J x_{\tau(n)}-J y_{\tau(n)}\right\rangle \\
& +\liminf _{n \rightarrow \infty}\left\langle y_{\tau(n)}-\Pi_{F} u, J \Pi_{F} u-J u\right\rangle .
\end{aligned}
$$

Since $J$ is uniformly continuous on bounded subsets of $E$ and $\left\|x_{\tau(n)}-y_{\tau(n)}\right\| \rightarrow 0$ in (3.6), we obtain

$$
\left.\| J x_{\tau(n)}-J y_{\tau(n)}\right\rangle \| \rightarrow 0,
$$

which implies

$$
\left|\left\langle y_{\tau(n)}-\Pi_{F} u, J x_{\tau(n)}-J y_{\tau(n)}\right\rangle\right| \leq\left\|y_{\tau(n)}-\Pi_{F} u\right\| \cdot\left\|J x_{\tau(n)}-J y_{\tau(n)}\right\| \rightarrow 0 .
$$


There exists a subsequence $\left\{y_{n_{i}}\right\}$ of $\left\{y_{\tau(n)}\right\}$ such that $y_{n_{i}} \rightarrow w \in F$ and

$$
\begin{aligned}
\liminf _{n \rightarrow \infty}\left\langle y_{\tau(n)}-\Pi_{F} u, J \Pi_{F} u-J u\right\rangle & =\lim _{n \rightarrow \infty}\left\langle y_{n_{i}}-\Pi_{F} u, J \Pi_{F} u-J u\right\rangle \\
& =\left\langle w-\Pi_{F} u, J \Pi_{F} u-J u\right\rangle \geq 0
\end{aligned}
$$

by Lemma 2.2. So, from (3.8), we get

$$
\limsup _{n \rightarrow \infty} \phi\left(\Pi_{F} u, y_{\tau(n)}\right)=0 .
$$

Since $\phi\left(\Pi_{F} u, x_{n}\right) \leq \phi\left(\Pi_{F} u, x_{\tau(n)+1}\right)$ for every $n \geq n_{0}$, by (3.3) we obtain

$$
\lim _{n \rightarrow \infty} \phi\left(\Pi_{F} u, x_{n}\right)=0 .
$$

This is a contradiction. So, $\left\{\phi\left(\Pi_{F} u, x_{n}\right)\right\}$ is convergent. Since $\left\{x_{n}\right\}$ and $\left\{y_{n}\right\}$ are bounded and $\gamma_{n} \rightarrow 0$, by (3.4) with $\beta=\beta_{0}$, we have

$$
\left\|x_{n}-y_{n}\right\| \rightarrow 0 \text { and }\left\|B x_{n}-B \Pi_{F} u\right\| \rightarrow 0 .
$$

By Lemma 3.2, we get $\omega_{w}\left(\left\{x_{n}\right\}\right) \subset F$. We show that

$$
\limsup _{n \rightarrow \infty}\left\langle\Pi_{F} u-y_{n}, J x_{n}-J u\right\rangle \geq 0 .
$$

Suppose that $\lim \sup _{n \rightarrow \infty}\left\langle\Pi_{F} u-y_{n}, J x_{n}-J u\right\rangle=l<0$. There exists $n_{1} \in \mathbb{N}$ such that

$$
\left\langle\Pi_{F} u-y_{n}, J x_{n}-J u\right\rangle \leq \frac{1}{2} l
$$

for every $n \geq n_{1}$. From (3.4) with $\beta=\beta_{0}$, we have

$$
|l| \gamma_{n} \leq 2 \gamma_{n}\left\langle y_{n}-\Pi_{F} u, J x_{n}-J u\right\rangle \leq \phi\left(\Pi_{F} u, x_{n}\right)-\phi\left(\Pi_{F} u, x_{n+1}\right)
$$

for each $n \geq n_{1}$, which implies

$$
\sum_{n=n_{1}}^{\infty}|l| \gamma_{n} \leq \phi\left(\Pi_{F} u, x_{n_{1}}\right)<\infty
$$

Since $\sum_{n=1}^{\infty} \gamma_{n}=\infty$, this is a contradiction. So, we get (3.9). Next, we have

$$
\begin{aligned}
\left\langle\Pi_{F} u-y_{n}, J x_{n}-J u\right\rangle= & \left\langle\Pi_{F} u-y_{n}, J x_{n}-J y_{n}\right\rangle+\left\langle\Pi_{F} u-y_{n}, J y_{n}-J \Pi_{F} u\right\rangle \\
& +\left\langle\Pi_{F} u-y_{n}, J \Pi_{F} u-J u\right\rangle \\
\leq & \left\|\Pi_{F} u-y_{n}\right\| \cdot\left\|J x_{n}-J y_{n}\right\|-\frac{1}{2} \phi\left(\Pi_{F} u, y_{n}\right) \\
& +\left\langle\Pi_{F} u-y_{n}, J \Pi_{F} u-J u\right\rangle
\end{aligned}
$$

for all $n \in \mathbb{N}$. Since $J$ is uniformly continuous on bounded subsets of $E$ and $\left\|x_{n}-y_{n}\right\| \rightarrow 0$, we have

$$
\left\|J x_{n}-J y_{n}\right\| \rightarrow 0 .
$$

So, we obtain

$$
\begin{aligned}
0 & \leq \limsup _{n \rightarrow \infty}\left\langle\Pi_{F} u-y_{n}, J x_{n}-J u\right\rangle \\
& \leq-\frac{1}{2} \liminf _{n \rightarrow \infty} \phi\left(\Pi_{F} u, y_{n}\right)+\limsup _{n \rightarrow \infty}\left\langle\Pi_{F} u-y_{n}, J \Pi_{F} u-J u\right\rangle .
\end{aligned}
$$


There exists a subsequence $\left\{y_{n_{j}}\right\}$ of $\left\{y_{n}\right\}$ such that $y_{n_{j}} \rightarrow w \in F$ and

$$
\begin{aligned}
\limsup _{n \rightarrow \infty}\left\langle\Pi_{F} u-y_{n}, J \Pi_{F} u-J u\right\rangle & =\lim _{j \rightarrow \infty}\left\langle\Pi_{F} u-y_{n_{j}}, J \Pi_{F} u-J u\right\rangle \\
& =\left\langle\Pi_{F} u-w, J \Pi_{F} u-J u\right\rangle \leq 0
\end{aligned}
$$

from Lemma 2.2. So, we have

$$
\liminf _{n \rightarrow \infty} \phi\left(\Pi_{F} u, y_{n}\right)=0 .
$$

By (3.3), we get $\liminf _{n \rightarrow \infty} \phi\left(\Pi_{F} u, x_{n+1}\right)=0$, that is, $\left\{x_{n}\right\}$ converges strongly to $\Pi_{F} u$ from Lemma 2.1.

By Theorem 3.1, we have the following new result in real Hilbert spaces. This holds under weaker conditions than the result in [30].

Theorem 3.2. Let $C$ be a nonempty closed convex subset of a real Hilbert space $H$. Let $A$ be a maximal monotone operator in $H \times H, \alpha>0, B$ an $\alpha$-inverse-strongly-monotone operator of $C$ into $H$ such that $F=(A+B)^{-1} 0 \neq \emptyset$. Let $u \in H$ and $\left\{x_{n}\right\}$ be a sequence generated by

$$
x_{1} \in C, \quad x_{n+1}=P_{C} J_{\lambda_{n}}^{A}\left(\gamma_{n} u+\left(1-\gamma_{n}\right) x_{n}-\lambda_{n} B x_{n}\right)
$$

for each $n \in \mathbb{N}$, where $P_{C}$ is the metric projection of $H$ onto $C,\left\{\lambda_{n}\right\} \subset(0, \infty)$ and $\left\{\gamma_{n}\right\} \subset(0,1]$ such that $\gamma_{n} \rightarrow 0$ and $\sum_{n=1}^{\infty} \gamma_{n}=\infty$. Then, if $0<\inf _{n \in \mathbb{N}} \lambda_{n} \leq \sup _{n \in \mathbb{N}} \lambda_{n}<2 \alpha$, then $\left\{x_{n}\right\}$ converges strongly to $P_{F} u$.

\section{DeducED RESUlts}

Let $C$ be a nonempty closed convex subset of $E$ and $A$ a single valued monotone operator of $C$ into $E^{*}$, that is, $\langle x-y, A x-A y\rangle \geq 0$ for all $x, y \in C$. We consider the variational inequality problem [19] for $A$, that is, the problem of finding an element $z \in C$ such that

$$
\langle x-z, A z\rangle \geq 0 \text { for all } x \in C .
$$

The set of all solutions of the variational inequality problem for $A$ is denoted by $\operatorname{VI}(C, A)$. By Theorem 3.1, we obtain a new result for the variational inequality problem of an inverse-strongly-monotone operator in a 2-uniformly convex and uniformly smooth Banach space $E$.

Theorem 4.1. Let $C$ be a nonempty closed convex subset of a 2-uniformly convex and uniformly smooth Banach space E. For $\alpha>0$, let $B$ be an $\alpha$-inverse-strongly-monotone operator of $C$ into $E^{*}$ with $V I(C, B) \neq \emptyset$. Let $u \in E$ and $\left\{x_{n}\right\}$ a sequence generated by $x_{1} \in C$ and

$$
x_{n+1}=\Pi_{C} J^{-1}\left(\gamma_{n} J u+\left(1-\gamma_{n}\right) J x_{n}-\lambda_{n} B x_{n}\right)
$$

for every $n \in \mathbb{N}$, where $\left\{\lambda_{n}\right\} \subset(0, \infty)$ and $\left\{\gamma_{n}\right\} \subset(0,1]$ are real sequences. Suppose that $0<\inf _{n \in \mathbb{N}} \lambda_{n} \leq$ $\sup _{n \in \mathbb{N}} \lambda_{n}<2 \alpha c$, where $c$ is the constant in Theorem 2.1, $\gamma_{n} \rightarrow 0$, and $\sum_{n=1}^{\infty} \gamma_{n}=\infty$. Then, $\left\{x_{n}\right\}$ converges strongly to $\Pi_{V I(C, B)} u$.

Proof. Let $i_{C}: E \rightarrow(-\infty, \infty]$ be the indicator function of $C$. We know that $i_{C}$ is proper lower semicontinuous and convex, and hence its subdifferential $\partial i_{C}$ is a maximal monotone operator. Let $A=\partial i_{C}$. Then, it is easy to see that $J_{\lambda} x=\Pi_{C} x$ for every $\lambda>0$ and $x \in E$, where $J_{\lambda}$ is the resolvent of $A$. Further, we also get $(A+B)^{-1} 0=V I(C, B)$. Hence the proof is complete. 
Remark 4.1. Using Theorem 4.1, we get a result for the variational inequality problem for a finite family of inverse-strongly-monotone operators as follows: Let $C$ be a nonempty closed convex subset of $E, r \in \mathbb{N}$ and $B_{i}$ a $\beta_{i}$-inverse-strongly-monotone operator of $C$ into $E^{*}$ for $i=1,2, \ldots, r$. Suppose $\bigcap_{i=1}^{r} V I\left(C, B_{i}\right) \neq \emptyset$ and let $A x=\frac{1}{r}\left(B_{1} x+B_{2} x+\cdots+B_{r} x\right)$ for every $x \in C$. Then, we have $A$ is also inversestrongly-monotone and $V I(C, A)=\bigcap_{i=1}^{r} V I\left(C, B_{i}\right)$. Indeed, for $x, y \in C$ and $\alpha=\min \left\{\beta_{1}, \beta_{2}, \ldots, \beta_{r}\right\}$, we get

$$
\begin{aligned}
& \langle x-y, A x-A y\rangle \\
& =\frac{1}{r}\left(\left\langle x-y, B_{1} x-B_{1} y\right\rangle+\left\langle x-y, B_{2} x-B_{2} y\right\rangle+\cdots+\left\langle x-y, B_{r} x-B_{r} y\right\rangle\right) \\
& \geq \frac{1}{r}\left(\beta_{1}\left\|B_{1} x-B_{1} y\right\|^{2}+\beta_{2}\left\|B_{2} x-B_{2} y\right\|^{2}+\cdots+\beta_{r}\left\|B_{r} x-B_{r} y\right\|^{2}\right) \\
& \geq \alpha\left(\frac{1}{r}\left\|B_{1} x-B_{1} y\right\|^{2}+\frac{1}{r}\left\|B_{2} x-B_{2} y\right\|^{2}+\cdots+\frac{1}{r}\left\|B_{r} x-B_{r} y\right\|^{2}\right) \\
& \geq \alpha\left\|\frac{1}{r}\left(B_{1} x-B_{1} y\right)+\frac{1}{r}\left(B_{2} x-B_{2} y\right)+\cdots+\frac{1}{r}\left(B_{r} x-B_{r} y\right)\right\|^{2} \\
& =\alpha\|A x-A y\|^{2} .
\end{aligned}
$$

Thus $A$ is an $\alpha$-inverse strongly monotone operator. Next, we show

$$
V I(C, A)=\bigcap_{i=1}^{r} V I\left(C, B_{i}\right) .
$$

The inclusion $V I(C, A) \supset \bigcap_{i=1}^{r} V I\left(C, B_{i}\right)$ is trivial. Let $u \in V I(C, A)$ and $z \in \bigcap_{i=1}^{r} V I\left(C, B_{i}\right)$. We have $\langle z-u, A u\rangle \geq 0$ and since $\left\langle u-z, B_{i} z\right\rangle \geq 0$ for all $i=1,2, \ldots, r$, we also get

$$
\langle u-z, A z\rangle=\frac{1}{r} \sum_{i=1}^{r}\left\langle u-z, B_{i} z\right\rangle \geq 0 .
$$

It follows that

$$
\langle z-u, A u-A z\rangle=\langle z-u, A u\rangle+\langle u-z, A z\rangle \geq \frac{1}{r} \sum_{i=1}^{r}\left\langle u-z, B_{i} z\right\rangle \geq 0 .
$$

On the other hand, we have

$$
\langle z-u, A u-A z\rangle \leq-\frac{\alpha}{r} \sum_{i=1}^{r}\left\|B_{i} z-B_{i} u\right\|^{2} \leq 0 .
$$

Therefore, we obtain

$$
B_{i} z=B_{i} u \text { and }\left\langle u-z, B_{i} z\right\rangle=0 \text { for all } i=1,2, \ldots, r .
$$

Hence we have

$$
\left\langle x-u, B_{i} u\right\rangle=\left\langle x, B_{i} z\right\rangle-\left\langle u, B_{i} z\right\rangle=\left\langle x, B_{i} z\right\rangle-\left\langle z, B_{i} z\right\rangle=\left\langle x-z, B_{i} z\right\rangle \geq 0
$$

for every $i=1,2, \ldots, r$ and $x \in C$, that is, $u \in V I\left(C, B_{i}\right)$ for all $i=1,2, \ldots, r$. Therefore, $V I(C, A) \subset$ $\bigcap_{i=1}^{r} \operatorname{VI}\left(C, B_{i}\right)$.

Remark 4.2. In the result of Iiduka and Takahashi [15], under the assumption that (i) $\|B y\| \leq\|B y-B u\|$ for every $y \in C$ and $u \in V I(C, A)$, and (ii) $J$ is weakly sequentially continuous, they proved the weak convergence of the generated sequence to an element of $V I(C, B)$, whereas we get the strong convergence to an element of $V I(C, B)$ in Theorem 4.1, without the assumptions (i) and (ii); see also [18]. 
Remark 4.3. In Lemma 3.2, if $A=0$, then, $J_{\lambda}^{A}=I$ for every $\lambda>0$. Moreover, we can get the inclusion $\omega_{w}\left(\left\{x_{n}\right\}\right) \subset B^{-1} 0$ from the assumption $\left\|B x_{n}-B z\right\| \rightarrow 0$ for $z \in B^{-1} 0$ only. In fact, suppose $x_{n_{i}} \rightarrow w \in C$. Since

$$
\left\langle x_{n_{i}}-w, B x_{n_{i}}-B w\right\rangle \geq \alpha\left\|B x_{n_{i}}-B w\right\|^{2}
$$

for every $i \in \mathbb{N}$, we have $B w=B z=0$, that is, $w \in B^{-1} 0$. Therefore, instead of uniform smoothness of $E$, smoothness of $E$ guarantees that Lemma 3.2 holds. Hence we obtain the following strong convergence theorem for monotone inclusions by Theorem 3.1. This is a result of strong convergence under weaker conditions than that of weak convergence in [15].

Theorem 4.2. Let $C$ be a nonempty closed convex subset of a 2-uniformly convex and smooth Banach space $E$. For $\alpha>0$, let $B$ be an $\alpha$-inverse-strongly-monotone operator of $C$ into $E^{*}$ with $B^{-1} 0 \neq \emptyset$. Let $u \in E$ and $\left\{x_{n}\right\}$ a sequence generated by $x_{1} \in C$ and

$$
x_{n+1}=\Pi_{C} J^{-1}\left(\gamma_{n} J u+\left(1-\gamma_{n}\right) J x_{n}-\lambda_{n} B x_{n}\right)
$$

for every $n \in \mathbb{N}$, where $\left\{\lambda_{n}\right\} \subset(0, \infty)$ and $\left\{\gamma_{n}\right\} \subset(0,1]$ are real sequences. Suppose that $0<\inf _{n \in \mathbb{N}} \lambda_{n} \leq$ $\sup _{n \in \mathbb{N}} \lambda_{n}<2 \alpha c$, where $c$ is the constant in Theorem 2.1, $\gamma_{n} \rightarrow 0$, and $\sum_{n=1}^{\infty} \gamma_{n}=\infty$. Then, $\left\{x_{n}\right\}$ converges strongly to $\Pi_{B^{-1} 0} u$.

\section{Acknowledgment}

This paper was supported by JSPS KAKENHI Grant No. 15K05007.

\section{REFERENCES}

[1] Y.I. Alber, Metric and generalized projection operators in Banach spaces: properties and applications. In: Kartosator, A.G. (ed.) Theory and Applications of Nonlinear Operators of Accretive and Monotone Type, pp. 15-50. Dekker, New York (1996).

[2] Y.I. Alber, S. Reich, An iterative method for solving a class of nonlinear operator equations in Banach spaces, Panamer. Math. J. 4 (1994), 39-54.

[3] K. Aoyama, Y. Kimura, F. Kohsaka, Strong convergence theorems for strongly relatively nonexpansive sequences and applications, J. Nonlinear Anal. Optim. 3 (2012), 67-77.

[4] H. Attouch, J. Peypouquet, P. Redont, Backward-forward algorithms for structured monotone inclusions in Hilbert spaces, J. Math. Anal. Appl. 457 (2018), 1095-1117.

[5] J.B. Baillon, G. Haddad, Quelques propriétés des opérateurs angle-bornés et n-cycliquement monotones, Israel J. Math. 26 (1977), 137-150.

[6] V. Barbu, Nonlinear semigroups and differential equations in Banach spaces, Editura Academiei R. S. R. Bucuresti, Romania, 1976.

[7] O.A. Boikanyo, The viscosity approximation forward-backward splitting method for zeros of the sum of monotone operators, Abstr. Appl. Anal. 2016 (2016), Article ID 2371857.

[8] F.E. Browder, Nonlinear maximal monotone operators in Banach spaces, Math. Ann. 175 (1968), 89-113.

[9] G.H-G. Chen, R.T. Rockafellar, Convergence rates in forward-backward splitting, SIAM J. Optim. 7 (1997), $421-444$.

[10] S.Y. Cho, X. Qin, L. Wang, Strong convergtence of a splitting algorithm for treating monotone operators, Fixed Point Theory Appl. 2014 (2014), Article ID 94.

[11] J. C. Dunn, Convexity, monotonicity, and gradient processes in Hilbert space, J. Math. Anal. Appl. 53 (1976), 145-158.

[12] D. Gabay, Applications of the method of multipliers to variational inequalities, in Augmented Lagrangian Methods: Applications to the Numerical Solution of Boundary-Value Problems (M.Fortin and R.Glowinski Eds.), Studies in Mathematics and Its Applications, North Holland, Amsterdam, Holland, Vol. 15, 299-331, 1983.

[13] B. Halpern, Fixed points of nonexpanding maps, Bull. Amer. Math. Soc. 73 (1967), 957-961. 
[14] Y. Haugazeau, Sur les inéquations variationnelles et la minimisation de fonctionnelles convexes, Thèse, Université de Paris, Paris, France, 1968.

[15] H. Iiduka, W. Takahashi, Weak convergence of a projection algorithm for variational inequalities in a Banach space, J. Math. Anal. Appl. 339 (2008), 668-679.

[16] S. Kamimura, W. Takahashi, Strong convergence of a proximal-type algorithm in a Banach space, SIAM J. Optim. 13 (2002), 938-945.

[17] Y. Kimura, K. Nakajo, The problem of image recovery by the metric projections in Banach spaces, Abstr. Appl. Anal. 2013 (2013), Article ID 817392.

[18] Y. Kimura, K. Nakajo, Strong convergence to a solution of a variational inequality problem in Banach spaces, J. Appl. Math. 2014 (2014), Article ID 346517.

[19] J.L. Lions, G. Stampacchia, Variational inequalities, Comm. Pure Appl. Math. 20 (1967), 493-517.

[20] P.L. Lions, B. Mercier, Splitting algorithms for the sum of two nonlinear operators, SIAM J. Numer. Anal. 16 (1979), 964-979.

[21] F. Liu, M.Z. Nashed, Regularization of nonlinear ill-posed variational inequalities and convergence rates, Set-Valued Anal. 6 (1998), 313-344.

[22] P.E. Maingé, Strong convergence of projected subgradient methods for nonsmooth and nonstrictly convex minimization, Set-Valued Anal. 16 (2008), 899-912.

[23] S. Matsushita, W. Takahashi, A strong convergence theorem for relatively nonexpansive mappings in a Banach space, J. Approx. Theory 134 (2005), 257-266.

[24] S. Matsushita, K. Nakajo, W. Takahashi, Strong convergence theorems obtained by a generalized projections hybrid method for families of mappings in Banach spaces, Nonlinear Anal. 73 (2010), 1466-1480.

[25] A. Moudafi, Viscosity approximation methods for fixed-point problems, J. Math. Anal. Appl. 241 (2000), 46-55.

[26] A. Moudafi, M. Théra, Finding a zero of the sum of two maximal monotone operators, J. Optim. Theory Appl. 94 (1997), 425-448.

[27] A. Moudafi, M. Oliny, Convergence of a splitting inertial proximal method for monotone operators, J. Comput. Appl. Math. 155 (2003), 447-454.

[28] K. Nakajo, W. Takahashi, Strong and weak convergence theorems by an improved splitting method, Commun. Appl. Nonlinear Anal. 9 (2002), 99-107.

[29] K. Nakajo, K. Shimoji, W. Takahashi, Strong convergence theorems by the hybrid method for families of nonexpansive mappings in Hilbert spaces, Taiwanese J. Math. 10 (2006), 339-360.

[30] K. Nakajo, K. Shimoji, W. Takahashi, Strong convergence theorems of Halpern's type for families of nonexpansive mappings in Hilbert spaces, Thai J. Math. 7 (2009), 49-67.

[31] G.B. Passty, Ergodic convergence to a zero of the sum of monotone operators in Hilbert space, J. Math. Anal. Appl. 72 (1979), 383-390.

[32] X. Qin, S.Y. Cho, L. Wang, A regularization method for treating zero points of the sum of two monotone operators, Fixed Point Theory Appl. 2014 (2014), Article ID 75.

[33] R.T. Rockafellar, Characterization of the subdifferentials of convex functions, Pacific J. Math. 17 (1966), 497-510.

[34] R.T. Rockafellar, On the maximal monotonicity of subdifferential mappings, Pacific J. Math. 33 (1970), 209-216.

[35] R.T. Rockafellar, On the maximality of sums of nonlinear monotone operators, Trans. Amer. Math. Soc. 149 (1970), 75-88.

[36] N. Shahzad, H. Zegeye, Approximating a common point of fixed points of a pseudocontractive mapping and zeros of sum of monotone mappings, Fixed Point Theory Appl. 2014 (2014), Article ID 85.

[37] M. Suwannaprapa, N. Petrot, S. Suantai, Weak convergence theorems for split feasibility problems on zeros of the sum of monotone operators and fixed point sets in Hilbert spaces, Fixed Point Theory Appl. 2017 (2017), Article ID 6.

[38] W. Takahashi, Nonlinear Functional Analysis, Yokohama Publishers, Yokohama, 2000.

[39] W. Takahashi, Convex Analysis and Approximation of Fixed Points, Yokohama Publishers, Yokohama, 2000 (Japanese).

[40] W. Takahashi, Y. Takeuchi, Y. Kubota, Strong convergence theorems by hybrid methods for families of nonexpansive mappings in Hilbert spaces, J. Math. Anal. Appl. 341 (2008), 276-286. 
[41] P. Tseng, A modified forward-backward splitting method for maximal monotone mappings, SIAM J. Control Optim. 38 (2000), 431-446.

[42] H.K. Xu, Inequalities in Banach spaces with applications, Nonlinear Anal. 16 (1991), 1127-1138.

[43] T. Yuying, S. Plubtieng, Strong convergence theorems by hybrid and shrinking projection methods for sums of two monotone operators, J. Inequal. Annal. 2017 (2017), Article ID 72.

[44] C. Zălinescu, On uniformly convex functions, J. Math. Anal. Appl. 95 (1983), 344-374.

[45] D.L. Zhu, P. Marcotte, Co-coercivity and its role in the convergence of iterative schemes for solving variational inequalities, SIAM J. Optim. 6 (1996), 714-726. 